\title{
Automated Collaborative Analysis System of Rockburst Mechanism based on Big Data
}

\author{
Yu Zhang ${ }^{\mathrm{a}, \mathrm{b}}$, Hongwei Ding ${ }^{\mathrm{a}, *}$, Yange Wang ${ }^{\mathrm{a}}$, Fuqiang Ren ${ }^{\mathrm{b}}$, Yongzhen Li ${ }^{\mathrm{a}}$, and Zhaoyong Lv ${ }^{\mathrm{a}}$ \\ ${ }^{a}$ School of Electrical and Information Engineering, Beijing University of Civil Engineering and Architecture, Beijing, 100044, China \\ ${ }^{b}$ State Key Laboratory in China for GeoMechanics and Deep Underground Engineering, China University of Mining and Technology, \\ Beijing, 100083, China
}

\begin{abstract}
In recent years, with the increase of the resource exploitation, mining depth is getting deeper and deeper. Meanwhile, the lives of mining workers have been threatened strongly. In order to reduce the probability of rockburst, researchers have carried out in-depth research on rockburst. He Manchao, the academician of the State Key Laboratory for GeoMechanics and Deep Underground Engineering, has initially simulated the occurrence of rockburst in the laboratory as well as studied the mechanism of rockburst. Because the amount of data accumulated in the experiment is as large as $1000 \mathrm{~T}$, using these valuable experimental data becomes a difficult problem. Therefore, we have introduced big data technology into the field of rockburst. We have designed and realized the automated collaborative analysis system of the rockburst mechanism based on big data. We have used acoustic emission sensors as data collection methods and selected the multi-task online learning algorithm for data processing and analyzing. We have achieved the selection of the inflection point in the process of force changes using Matlab. In addition, the inflection point to be checked in the system can obtain the corresponding part of analysis diagrams. The theoretical analyses and experimental studies show that the automated collaborative analysis system can have an obvious influence on rockburst data processing, which provides a good foundation for the study of the rockburst mechanism.
\end{abstract}

Keywords: rock burst; big data; visualization; online learning algorithm

(Submitted on April 12, 2018; Revised on May 29, 2018; Accepted on June 19, 2018)

(C) 2018 Totem Publisher, Inc. All rights reserved.

\section{Introduction}

\subsection{The significance of Rock Burst Research}

In general, rockburst is a rock explosion that occurs during the mining process, concretely explained as a sudden violent release of the elastic deformation potential energy accumulated in the rock mass, resulting in the rockburst and ejection. Rockbursts can happen suddenly or last for days to months. A rockburst accident occurred in the Leipzig coal mine in the 1730s and was the earliest rockburst ever recorded in the world [7]. Since then, this phenomenon has attracted people's attention. With the development of human society, the demand for resources is greatly increased, the depth of mining is increasing, and the occurrence of rock explosion accidents is more and more frequent.

As a worldwide geological hazard, rockburst greatly endangers the safety of construction workers. Reducing this phenomenon is one of our research goals.

\subsection{Research Status}

In the research of rockburst, Jiangxi Polytechnic University and Shanghai Pengxu Information Technology Company used a tungsten mine as the engineering background. It is significant for the actual production to establish a grey catastrophe prediction model for rockburst [6]. In 2017, W. H. Zhu et al. performed a large-scale rock burst model test under different stress gradients by using a self-developed gas-liquid composite rock burst model test device [14]. A new method for

\footnotetext{
* Corresponding author.

E-mail address: myscholar@sina.com
} 
recording the stress state changes of submicron particles formed on the surface of the specimen under uniaxial compression is proposed by K. N. Trubetskoy et al. [10] A. Manouchehrian and M. Cai analyzed the rockburst of tunnels under static and dynamic loads [1]. B. G. He and R. Zelig studied rockburst in discontinuous rock masses using theoretical and numerical methods [5].

With the increasing attention to the field of rockburst, further improvement of experimental research on rockburst has produced more and more experimental data. The data volume has increased from GB level to TB and PB level. This large amount of data will be very difficult to solve using traditional technologies; therefore, we introduce big data technology into the field of rock burst.

\section{Big Data Technology}

In June 2015, President Xi emphasized the big data acquisition and application in China when he visited the Big Data Application Exhibition Center in Guizhou. There is no doubt that big data has a growing impact on the development of human society. With the fourth World Internet Conference held in Wuzhen, President Xi also proposed to promote the development of big data in his congratulatory letter [11].

Big data research mainly involves the collection, analysis, storage, management and security and privacy protection. H. Guo, J. Liu, and L. Zhao proposed the technology of the large optical wireless data acquisition problem to enhance the smart grid fault [4]. Y. Fu et al. performed a big data analysis of the anti-APT attack defense system framework and intelligent feedback system security testing framework [3]. K. Raichura and N. Padhariya proposed a BigCache architecture in mobile network data management [9]. A.S. Panah, R.V. Schyndel, and T. Sellis proposed asynchronous convergence watermark technology for big data end-to-end protection [8]. There are many researchers carrying out relevant research in the field of big data.

To provide good data for the development of the environment at the national and social levels, we have to make big data service in our production and life. Early big data technology is mainly used in commercial, financial, and other fields. It has gradually extended to medical, transportation, energy, and other fields. Through the automated collaborative analysis system of the rockburst mechanism based on big data, we will introduce the observation and prevention in rockburst.

\section{Architecture}

In order to make big data technology serve this research best, we designed and implemented the automated collaborative analysis system of the rockburst mechanism based on big data. The architecture of the collaborative analysis system is mainly composed of five parts: big data acquisition, big data storage, information service, on-line analysis, and visualization, as shown in Figure 1.

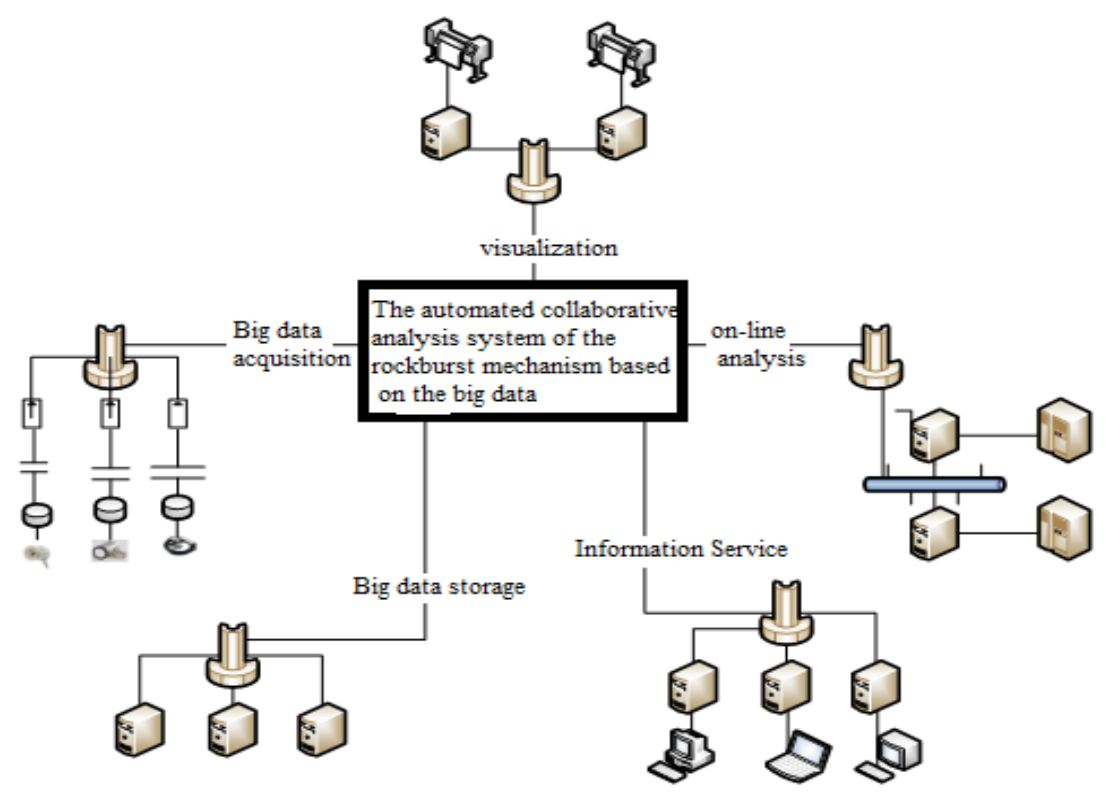

Figure 1. The figure of the automated collaborative analysis system 


\subsection{Big Data Acquisition Subsystem}

In the big data acquisition module, when we collect rockburst data, we use the technology of acoustic emission, which can be applied to many engineering fields, such as coal mining, pipeline system testing, railway system testing, and aviation maintenance $[2,13,15]$. Since Obert and Duvall discovered the phenomenon of acoustic emission in rocks in the 1930s, acoustic emission technology has been widely applied to monitor the characteristics of acoustic emission in rocks. This is shown in Figure 2.

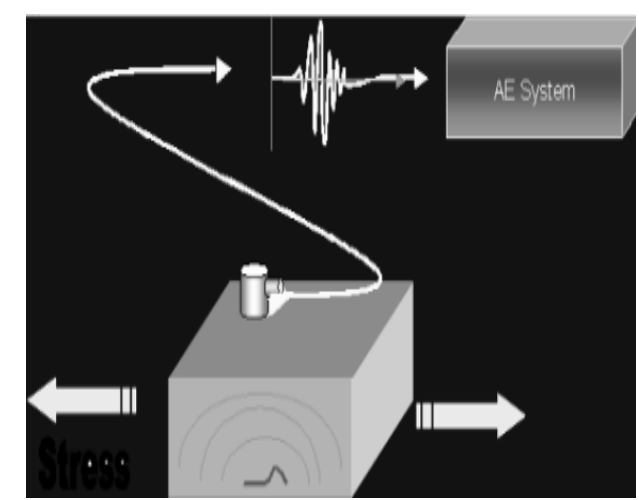

Figure 2. The principle model of acoustic emission detection

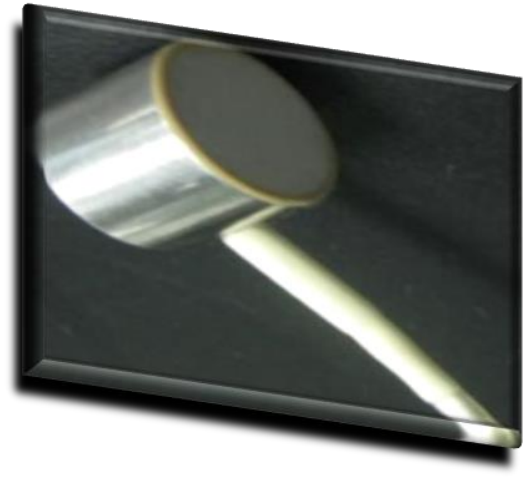

Figure 3. Acoustic emission sensor

Granite is used as an experimental sample. Through the process of exerting different stresses and removing stresses, the waveform of the rock is transmitted to the amplifier through the sensor, and then it is processed by the signal processing device to form the map of the intrinsic spectrum of granite. Acoustic emission detection requires the conversion of acoustic emission signals into electrical signals through sensors. Here, we choose a professional acoustic emission sensor, such as in Figure 3.

The data acquisition part collects ten sets of experimental data from granite. Here, we select five groups of experimental results for reference.

Table 1. Data summary of granite rock burst experimental data

\begin{tabular}{|c|c|c|c|}
\hline Experimental number & Generating the number of TXT & $\begin{array}{c}\text { The number of } \\
\text { experimental values }\end{array}$ & $\begin{array}{c}\text { Occupied space } \\
\text { (GB })\end{array}$ \\
\hline $61 \#$ & 13735 & 56258560 & 0.701 \\
\hline $62 \#$ & 248488 & 1017806848 & 12.3 \\
\hline $65 \#$ & 172585 & 706908160 & 17.2 \\
\hline $68 \#$ & 289443 & 1185558528 & 4.02 \\
\hline $70 \#$ & 230542 & 944300032 & 11.4 \\
\hline
\end{tabular}

Table 1 only shows the results of five sets of experiments. The State Key Laboratory of Deep Geotechnical and Underground Engineering has accumulated many experiments and data, and processing is much more difficult.

\subsection{Big Data Storage Subsystem}

After data acquisition, we first need to do a lossless compression of raw data. We need to ensure that data is not lost in the compressed storage process. At the same time, we need to try to reduce the amount of data occupied by the data. Data storage is detailed in the article of our research group members.

\subsection{Information Query Subsystem}

After the experimental data is uploaded to the system, we can also query all the uploaded data. We can query according to the time sequence of upload, the author's name, and the file size. The system calls the metadata to the query module through the user's request. The specific query method is no longer described here.

\section{System Implementation and Experimental Analysis}

\subsection{System Realization Effect}

The system of the first TXT 61\# experiment analysis results are shown separately in Figure 4, respectively, (1) The timedomain waveform; (2) 2-D spectrum; (3) The contour map; (4) The 3D graph. 


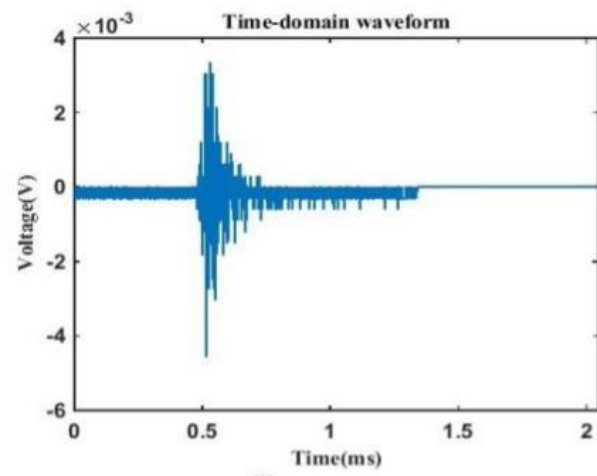

(1)

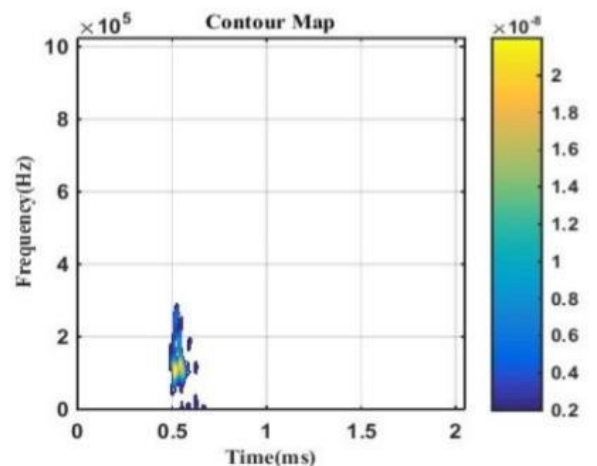

(3)

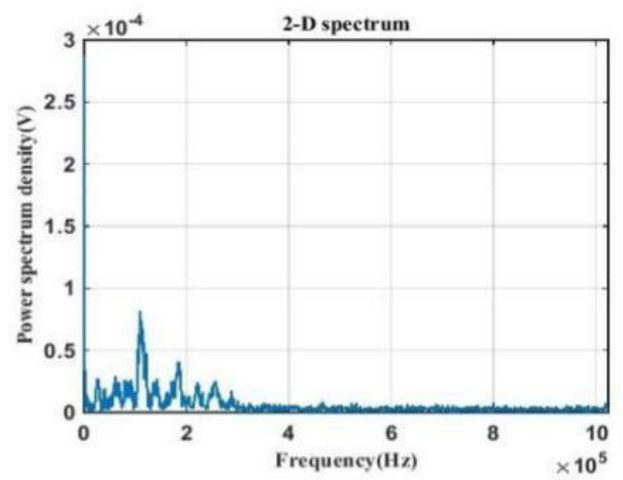

(2)

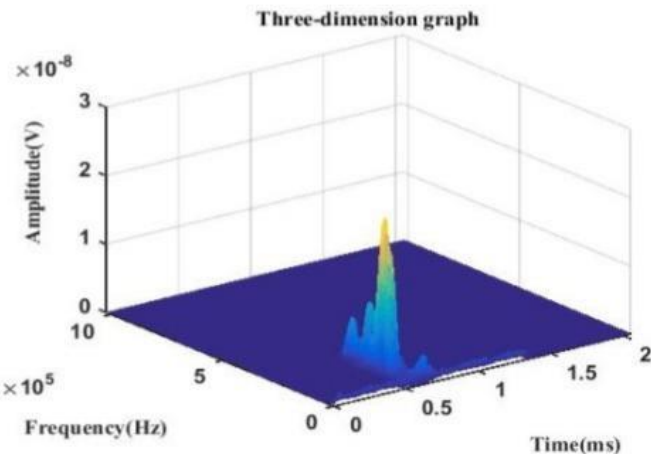

(4)

Figure 4. Local analysis diagram

Analysis of the entire experimental process of 61\# is shown in Figure 5-8. All 13,735 txts from this set of experiments are analyzed, which is an impossible task without the introduction of big data technology.

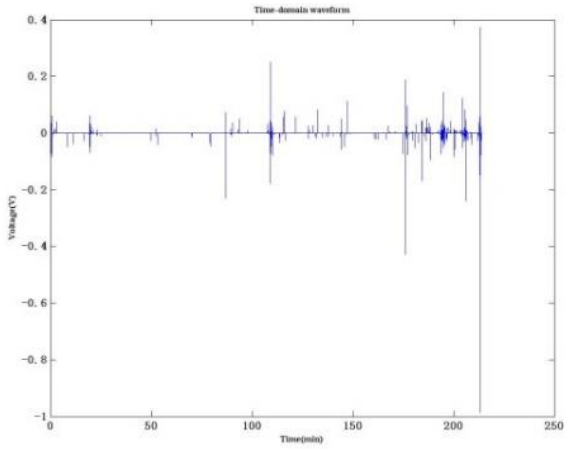

Figure 5. Time waveform global diagram

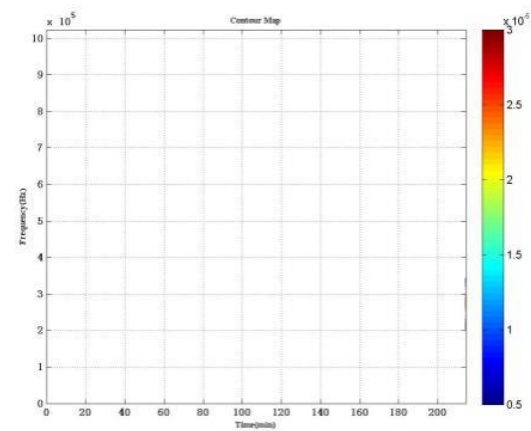

Figure 7. Time-frequency global diagram

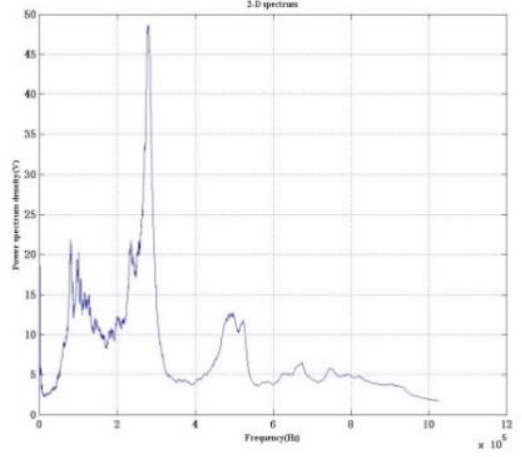

Figure 6. Spectrum global view

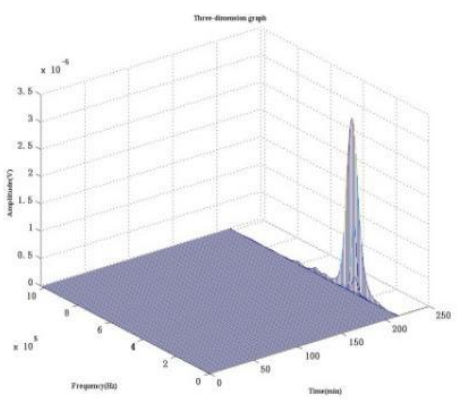

Figure 8. Three-dimensional global diagram 


\subsection{On-line Analysis and Visualization}

For real-time transmission of experimental data, in order to maintain its storage and analysis at the same time, we use the technology of online analysis. The online learning method adopts the model of direct data flow processing, which can effectively balance the real-time and accuracy and is suitable for the training of large flow data. In our system, the multitask online learning algorithm is used, and the pseudocode of the algorithm is described as follows [12]:

Input: $W_{0}=\arg _{W} \operatorname{minh}(W), \quad(\mathrm{W}$ is the weight matrix of the model $)$

$h(\mathrm{w})=\frac{1}{2}\|w\|_{F}^{2}$ Given constant $\lambda>0, \gamma>0$;

Initialization: $W_{1}=W_{0}, \overline{G_{0}}=0$.

for $\mathrm{t}=1,2,3, \ldots$, do

An instance of a $\mathrm{Q}$ task appears in turn $z_{t}^{q}, \quad \mathrm{q}=1, \ldots, \mathrm{Q}$, Calculated separately $g_{t}^{q}=\partial l_{t}\left(w_{q}, z_{q}^{t}\right)$, Form a matrix $\left.\mathrm{d} \times \mathrm{Q}, G_{t}=g_{t}^{1}, \ldots, g_{t}^{Q}\right) ;$

(1) Calculate the average gradient

$\overline{G_{t}}=\frac{t-1}{t} \overline{G_{t-1}}+\frac{1}{t} G_{t}$;

(2) Update the weight matrix of the next iteration based on $\mathrm{t} \bar{G}_{\mathrm{t}} W_{t+1}$,

$W_{t+1}=\arg _{W} \min _{\varphi}(W)$,

$\varphi(\mathrm{W})=\left(\overline{G_{t}}, W\right)+\Omega_{\lambda}(W)+\frac{\gamma}{\sqrt{t}} h(W)$.

end for

$\|\cdot\|_{F}$ denotes the norm of the matrix, regularization term $\Omega_{\lambda}(W)$ take weight matrix W to mix norm $L_{1 / 2,1}$, it's consists of $\left\|W_{j}^{T}\right\|_{1}$ and $\left\|W_{j}^{T}\right\|_{2}$, the norm of $L_{1,1}$ and the norm of $L_{2,1}$,

$\Omega_{\lambda}=\lambda \sum_{j=1}^{d}\left(c\left\|W_{j}^{T}\right\|_{1}+\left\|W_{j}^{T}\right\|_{2}\right.$ (c is constant)

In order to analyze the inflection point characteristics of the experimental data, we analyzed the data characteristics according to the multitask online learning algorithm, and the structure is shown in Figure 9.

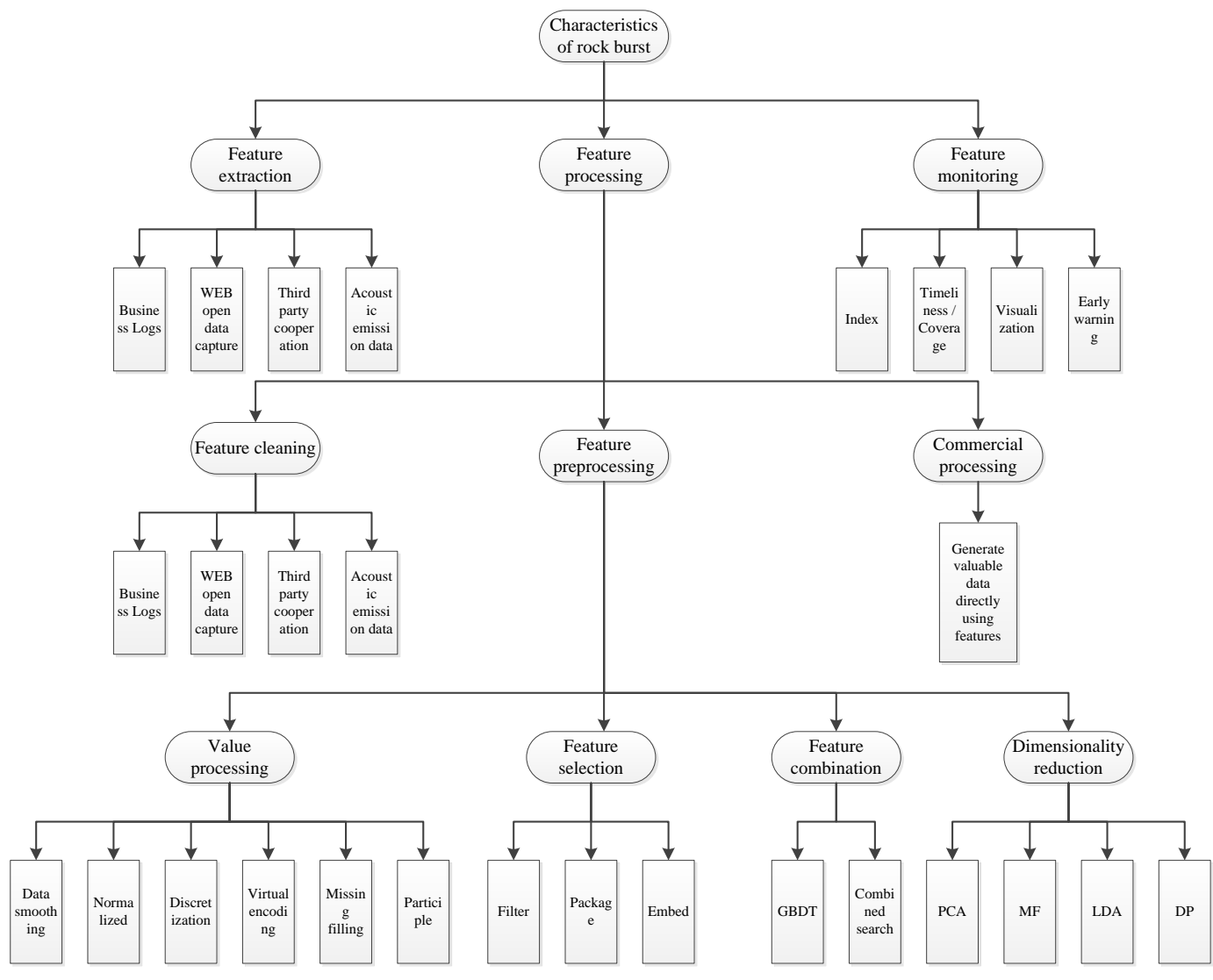

Figure 9. Rockburst data feature analysis architecture 


\subsection{Matlab Concrete Realization of the Algorithm Selection Inflection Point}

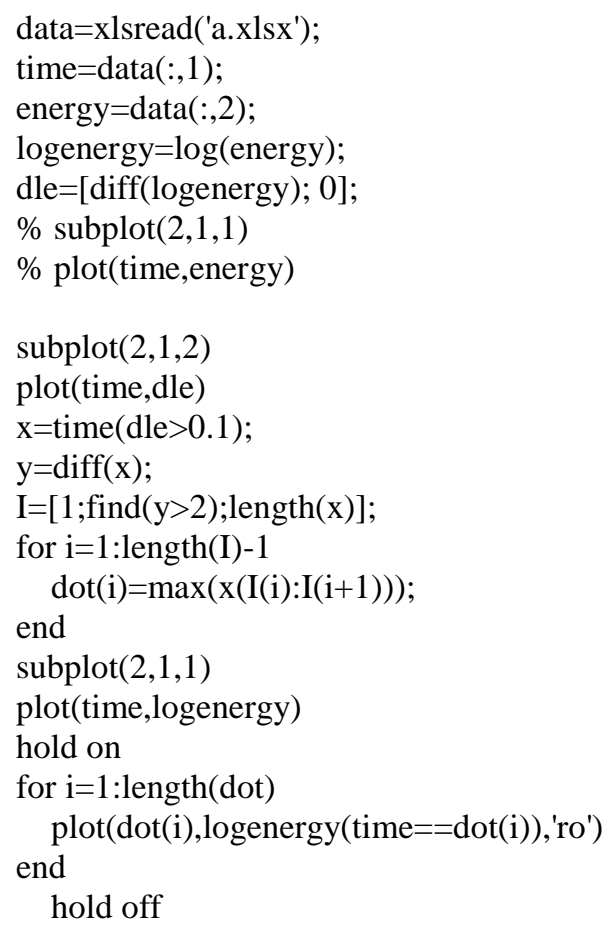

\subsection{Visualization of Analysis}

The automated collaborative analysis system of the rockburst mechanism based on big data can make a linkage analysis of inflection points, as shown in Figure 10, specifically: a stress map, time domain waveform, frequency spectrum, and energy diagram.

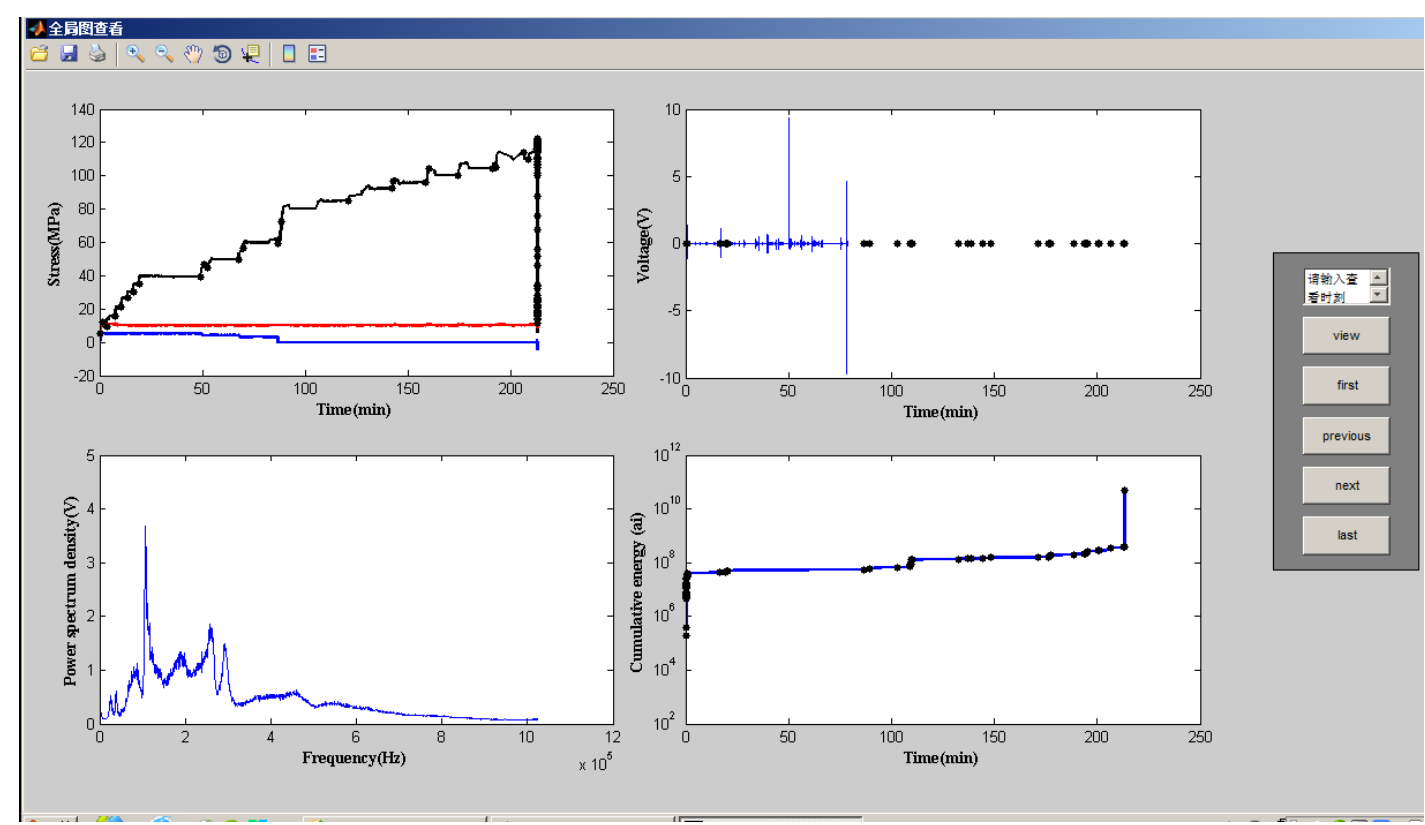

Figure 10. Linkage analysis of the initial diagram

For a selected stress diagram at a certain point in time, you can link the analysis of the corresponding data in the corresponding time analysis of the local diagram, as shown in Figure 11: 


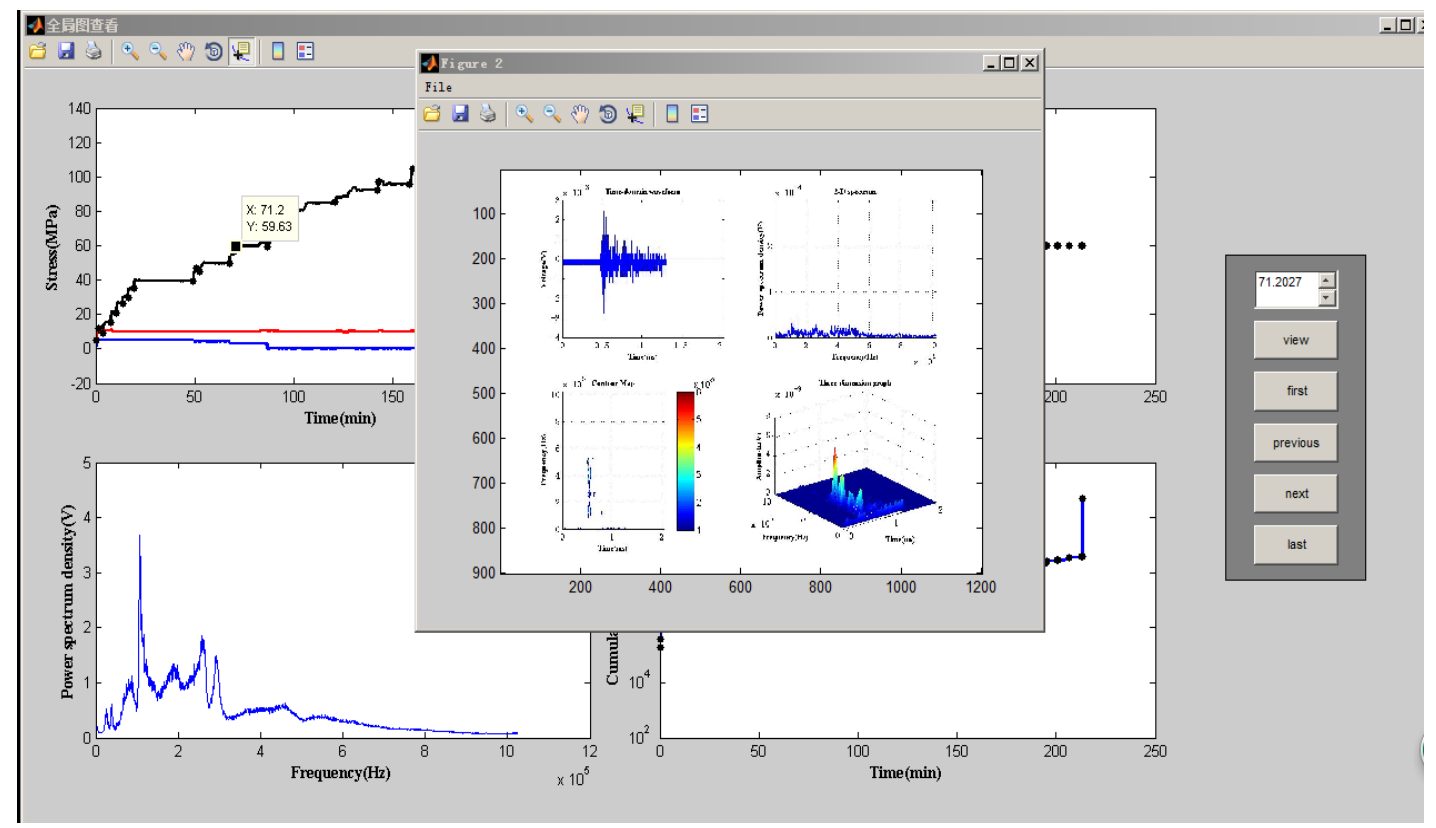

Figure 11. Linkage analysis results

When looking at the local analysis diagram at $71.2 \mathrm{~min}$, just enter the time value 71.2 you want to view into the box on the right and click on "view" to make a linkage with the big data analysis local diagram. It is convenient for us to view the time-domain waveform, frequency spectrum, time-frequency diagram, and 3D diagram of the inflection point on the stress diagram so that the rockburst characteristics of granite can be better analyzed.

\section{Conclusions}

Through the automated collaborative analysis system of the rockburst mechanism based on big data, we can deal with rockburst experimental data more efficiently. In addition to analyzing each txt separately in the experiment, this approach also analyzes the whole experiment process. At the same time, according to the parameter files and global diagrams, we can see the corresponding large-scale data analysis under stress time, diagram, which is impossible. At present, big data technology and online learning algorithms have been incorporated into the system. Our next goal is to make further analyses and apply time series analysis to dynamic data processing. In order to achieve better results on this basis, we must complete a more comprehensive study.

\section{Acknowledgments}

This work was partly supported by the National Key R\&D Program of China (No. 2016YFC060090) the Fundamental Research Funds for Beijing Universities response by zhangyu, the Foundation of the State Key Laboratory for GeoMechanics and Deep Underground Engineering (No. SKLGDUEK1523), the Foundation of the Beijing University of Civil Engineering and Architecture (No. 00331615029), the Excellent Teachers Development Foundation of BUCEA (No. 21082717046), the Institute of Energy and Environmental Sciences of the Beijing Coal Mine Innovation Alliance (No. 00362016055), and the graduate student innovation project of the Beijing University of Civil Engineering and Architecture (No. PG2017021).

\section{References}

1. M. Amin, and M. Cai, "Analysis of Rockburst in Tunnels Subjected to Static and Dynamic Loads," Journal of Rock Mechanics and Geotechnical Engineering 9.6(2017):1031-1040

2. Y. T. Chen, J. Qin, J. G. Li et al, "Application of AE Technique in Coal and Gas Outburst Prediction," MINING SAFETY\&ENVIRONMENTAL PROTECTION, 2017,(06):1-5

3. Y. Fu, H. C. Li, X.P. Wu et al, "Detecting APT Attacks: A Survey from the Perspective of Big Data Analysis," Journal on Communications, 2015,36(11):1-14.

4. H. Guo, J. Liu, L. Zhao, “ Big Data Acquisition under Failures in FiWi Enhanced Smart Grid," IEEE Transactions on Emerging Topics in Computing, 2017, PP(99):1-1. 
5. B. G. He, Zelig. R, Y. H. Hatzor et al, " Rockburst Generation in Discontinuous Rock Masses,” Rock Mechanics \& Rock Engineering, 2016(10):1-22.

6. W. W. Jiang, Z. H. Su, X. X. Chen et al, "Stability Prediction Model of Rock Mass Based on Microseismic Monitoring System," MINING R\&D ,2016,36(11):41-44.

7. G. Li, C. J. Zhou, Y. Zhang et al, "Review of Rockburst in Underground Engineering," Advances in Science and Technology of Water Resources, ,2013,33(03):77-83+94

8. A. S. Panah, R. V. Schyndel, T. Sellis, “ Towards an Asynchronous Aggregation-capable Watermark for end-to-end Protection of Big Data Streams," Future Generation Computer Systems, 2017, 72:288-304.

9. K. Raichura, and N. Padhariya, "Big Cache: A Cache-based Big Data Management in Mobile Networks," International Journal of Mobile Communications, 2017, 15(1):49.

10. K. N. Trubetskoy, et al, "Rockburst Prediction based on Submicron Particle Emission in Rocks under Deformation and Failure," Gornyi Zhurnal 6(2017):16-20.

11. "Xi Jinping to the Fourth World Internet Conference Congratulation Letter," Available at http://www.chinanews.com, Last accessed on Dec 3, 2017

12. H. Yang, M. R. Lyu, I. King, “Efficient Online Learning for Multitask Feature Selection,” Acm Transactions on Knowledge Discovery from Data, 2013, 7(2):1-27

13. P. L. Zhang, R. J. Wang, Y. C. Jiang et al, “Acoustic Emission Technology Research of Pipeline Leak Detection,” Journal of Gansu Sciences, 2017,29(03):86-91.

14. W. H. Zhu, N. Ma, Y. Y. Xia et al, "Model Tests on Rock Burst Using Gas-liquid Composite Loading," Chinese Journal of Rock Mechanics and Engineering, 2017,36(01):159-166.

15. X. Zhang, Y. Wang, H. S. Hu et al, "Research and Application of Acoustic Emission Technique in Railway System Inspection," Journal of Applied Acoustics, 2017,36(03):189-199.

Yu Zhang received a doctor's degree in Computer Science \& Engineering at the Beijing Institute of Technology. He is currently an associate professor and master supervisor in the School of Electrical and Information Engineering at the Beijing University of Civil Engineering and Architecture. His research interests include big data, distributed networks, artificial intelligence, and rockburst.

Hongwei Ding received a Master's degree from the School of Electrical and Information Engineering at the Beijing University of Civil Engineering and Architecture. Her research interests include big data and rockburst.

Yange Wang received a Master's degree from the School of Electrical and Information Engineering at the Beijing University of Civil Engineering and Architecture. Her research interests include distributed networks and rockburst.

Fuqiang Re is a Ph.D. student at the China University of Mining and Technology in Beijing. His major is geotechnical engineering. His research interests are strain burst and impact burst mechanisms.

Yongzhen Li received a Master's degree in Computer Science \& Engineering at the Beijing Institute of Technology. He is currently studying for his doctor's degree at the Beijing University of Technology. His research interests include big data, virtualization, and code obfuscation.

Zhaoyong Lv received a Master's degree in Control Theory \& Engineering in March 2011 from the Beijing University of Civil Engineering and Architecture. He is currently an experiment teacher at the Computing Center. His research interests include big data and control algorithm. 